\title{
QUERCUS CORRUGATA HOOKER (FAGACEAE) COMO INDICADOR PALEOCLIMÁTICO DEL PLEISTOCENO DE COSTA RICA
}

\author{
Eduardo A. Pérez ${ }^{1}$ \\ César A. Laurito ${ }^{2}$ y 3 \\ ${ }^{1}$ Dirección de Geología, Ministerio de Ambiente y Energía, Apdo. Postal 62-1009, \\ San José Costa Rica. E-mail: eperez@costarricense.cr \\ 2 INA, Instituto Nacional de Aprendizaje, Núcleo de Turismo, \\ Proceso de Planeamiento y Evaluación \\ ${ }^{3}$ Centro Independiente de Investigaciones Paleontológicas y Geológicas, Apdo. \\ Postal 203-2200, Coronado; San José, Costa Rica. \\ E-mail:Cesar_laurito@ hotmail.com
}

(Recibido: 19/2/02; Aceptado: 4/7/03)

\begin{abstract}
The fossil acorns were collected from the travertine La Palmera near to San Carlos. Today, Quercus corrugata inhabits over 1200 meters above the sea level and the occurrence of this species at 400 meters confirms important decrease of the temperature during the Pleistocene. It seems to be corresponding with the maximal glacial moment between 50000 to 13000 years BP.

RESUMEN: Se describen 21 muestras de improntas de frutos fósiles de Quercus corrugata Hooker, procedentes de un yacimiento de travertino de la localidad de La Palmera de San Carlos, cuya altura se ubica entre los 200 y $400 \mathrm{~m}$ sobre el nivel del mar. Se asume una edad Pleistoceno Superior para este yacimiento, en vista de que se considera la máxima glacial, comprendido entre 50000 a 13000 años antes del presente, como el mecanismo causante de la migración de esta especie a tales alturas.
\end{abstract}

\section{INTRODUCCIÓN}

La localidad de La Palmera se ubica en el poblado del mismo nombre a $16 \mathrm{~km}$ al NW de ciudad Quesada, cantón de San Carlos en la provincia de Alajuela (Fig.1).

El afloramiento corresponde a una cantera de travertino, ubicada entre los 200 y $400 \mathrm{~m}$ de altura, que actualmente se explota para la producción de cal y carbonato de calcio. Aparte de una gran cantidad de frondas fósiles, se han encontrado en dicho afloramiento restos de vertebrados, artrópodos diplópodos, crustáceos decápodos y gasterópodos.

La flora fósil está constituida fundamentalmente por moldes de hojas y de manera 
excepcional se han preservado moldes de frutos con gran detalle anatómico, entre los que destacan las nueces de Quercus o robles de altura, objetos del presente estudio. El material descrito se encuentra depositado en la colección de fósiles de la Escuela Centroamericana de Geología de la Universidad de Costa Rica.

\section{GEOLOGÍA}

El afloramiento se asocia a un sistema de fallas escalonadas de rumbo NW-SE, paralelo al margen norte del volcán Platanar, por el cual emergen aguas termales enriquecidas en carbonato de calcio, el cual se asume proviene de la Formación Venado de edad Mioceno Medio.

$\mathrm{El}$ afloramiento, con una longitud visible estimada de 1,3 km y $200 \mathrm{~m}$ de ancho, se formó por depositación de lechos sucesivos de carbonato, llegando a alcanzar un espesor de alrededor de 40 metros. El contacto inferior de esta unidad

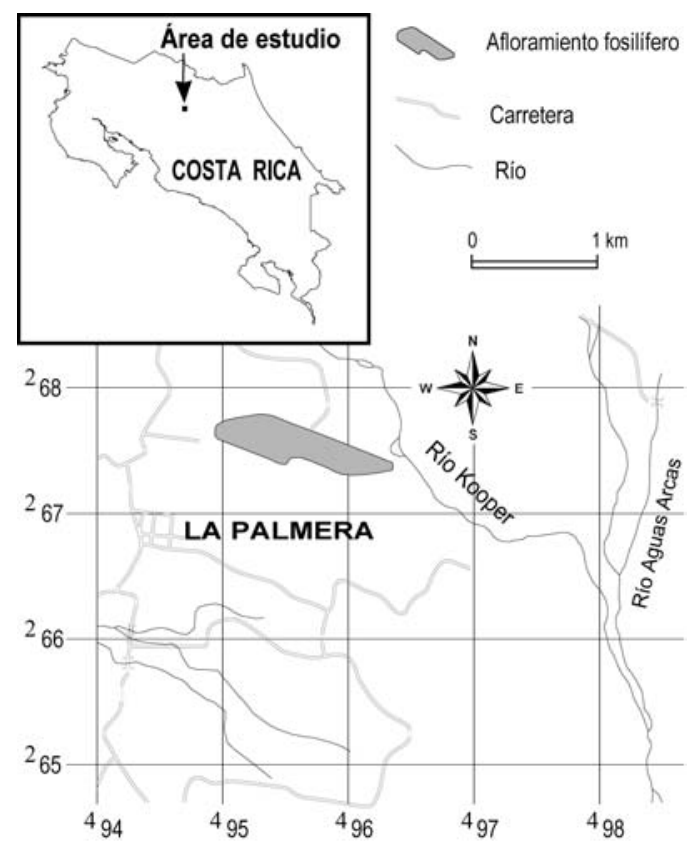

Fig. 1: Ubicación del afloramiento fosilífero de la Palmera de San Carlos, Mapa base: Hoja Aguas Zarcas del I.G.N. a escala 1:50 000 edición 2 (1986), digitalizó: C. Ramírez (DGM) no es visible, pero se presume que sobreyace depósitos piroclásticos diversos. Es sobreyacida por depósitos de cenizas, que en algunos puntos se interdigitan con los bancos de travertino como capas de tobas.

Actualmente, el proceso de formación de travertino y la fosilización de material vegetal continúa, lo que permitió evaluar las condiciones tafonómicas.

\section{TAFONOMÍA}

Una estación seca bien marcada es una de las condiciones necesarias para que ocurra el proceso de fosilización de la materia vegetal, debido a que la fijación del carbonato por la actividad de algas se incrementa al disminuir el caudal, lo que no permite a los restos vegetales (ramas, hojas y frutos) ser llevados por la corriente aguas abajo. Por otra parte, al disminuir el caudal, la temperatura se incrementa al igual que la concentración de carbonato, lo que optimiza el proceso de fosilización de las frondas.

La preservación de los frutos, también sugiere un sepultamiento, si no autóctono al menos parautóctono, ello debido a su excepcional preservación. A pesar de ser infrecuentes en relación con la cantidad de hojas registradas.

La escasez de frutos puede obedecer a varios factores, entre los más obvios están el ser una fuente de nutrientes, por lo que su aprovechamiento por otros organismos provoca una selección destructiva que imposibilita su registro fósil. Otro es la consistencia: frutos carnosos o tiernos tienen poca posibilidad de fosilización en relación con frutos duros como las nueces. En nuestro caso, las nueces de Quercus corrugata Hook, presentan condiciones ideales para su fosilización, e igualmente cuentan con un potencial de transporte muy grande. Sin embargo, la topografía en que se encuentra el afloramiento, sugiere que el transporte desde zonas alejadas no fue algo viable, ya que el depósito de travertino se encuentra en la cima de una colina.

Por otra parte, el registro de estos frutos constituye en sí mismo un evento extraordinario. La muestra de frutos fósiles de Quercus corrugata 
Hook es muy variada, incluye tanto frutos inmaduros como maduros y están limitados a una única capa de travertino ubicada en la parte media del afloramiento.

\section{SISTEMÁTICA}

\section{CLASE MAGNOLIOPSIDA \\ Subclase MAGNOLIIDAE Orden FAGALES \\ Familia FAGACEAE}

\section{Género Quercus}

El fruto es una bellota encerrada por una envoltura llamada involucro de la cúpula (distintiva del género) y desarrollándose a la madurez en 1 ó 2 años. Presenta forma ovoide a subglobosa o en espiral, achatado y con una cicatriz redonda en la base, el pericarpio es duro, glabro o con presencia de vellos blanquecinos en la superficie interior.

\section{Quercus corrugata Hooker}

Árboles de 6 a $20 \mathrm{~m}$ y tronco de $70 \mathrm{~cm}$ de diámetro, la corteza castaño oscuro se descascara en pedazos planos grandes, glabros o minuciosamente tomentoso-estrellado en los nodos, de color castaño pasando a pardo o pardo amarillento. Hojas deciduas, pecíolos cilíndricos o ligeramente surcado, glabros o muy detalladamente puberulento con vello simple, la forma de la hoja es estrechamente elíptica, con ápice acuminado o agudo, la base es abruptamente obtusa y ocasionalmente cordada o truncada, el margen es entero debajo del tercio o cuarto basal y con dientes serrados en el resto (raro entera a todo lo largo del margen), lámina glabra o con algunos largos vellos que persisten a lo largo de la vena media. Las espigas masculinas y flores femeninas no se han observado en Costa Rica. El fruto es solitario sobre un grueso pedúnculo de $4 \mathrm{~mm}$, la cúpula mide alrededor de $1,5 \mathrm{~cm}$ de largo y 3 $\mathrm{cm}$ de ancho, pero se ha dicho que llega a los 6 $\mathrm{cm}$ de ancho. Las escamas que componen la cú- pula son engrosadas basalmente y estrechamente apretadas, es densamente puberulento, la forma de la bellota es subglobosa a ovoide o cilíndrica (Fig. 2; a y b), con 2,5 cm de largo y $1,5 \mathrm{~cm}$ de grosor, pero se afirma que hay de 3 a $5 \mathrm{~cm}$ de grosor, la bellota es longitudinalmente lisa o corrugada con una marca o cicatriz en la base de 12 a $16 \mathrm{~mm}$ de diámetro, el área de secado sobre la cicatriz se contrae al secar.

Materiales estudiados: El código corresponde al listado de la colección de fósiles de la Escuela Centroamericana de Geología.

Muestra: CF 4598 (Fig. 3, a-e): La roca recolectada mide $60 \mathrm{~cm}$ de largo por $30 \mathrm{~cm}$ de ancho, presenta bastantes fragmentos de limbos incompletos con características de esta especie; se observan 7 moldes de frutos de Quercus corrugata, constituidos por improntas de nueces cilíndricas cuyos diámetros varían aproximadamente de $2,5 \mathrm{~cm}$ a $3,7 \mathrm{~cm}$. Además, se observan en cuatro de las muestras improntas de las cúpulas (Fig. 4, b y c) con escamas bien definidas.

Muestra: CF 4599 (Fig. 5, a-e): La roca recolectada mide $50 \mathrm{~cm}$ de largo por $30 \mathrm{~cm}$ de ancho, se observan bastantes moldes de limbos incompletos con las característica de esta especie. Se presentan 6 moldes de frutos de Quercus, constituidos por la impronta de una nuez y su cúpula. Los diámetros de las cúpulas varían de 4 a $4,5 \mathrm{~cm}$. En algunos ejemplares se registran las impresiones de las escamas y en dos de las muestras se observa el molde del pedúnculo (Fig. 6, a, b, c).
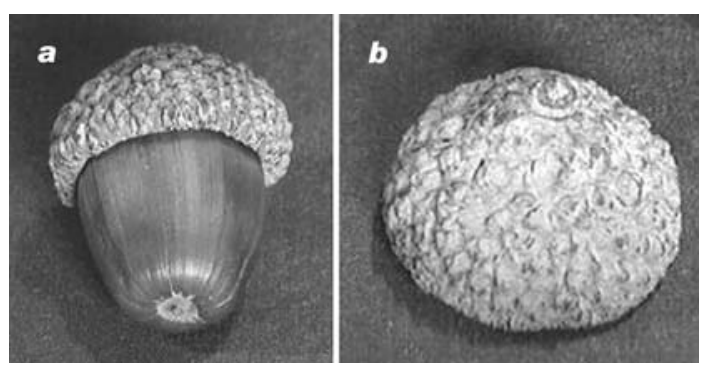

Fig. 2: Fruto actual de Quercus, se observa una bellota actual y detalle de la cúpula; la nuez mide $2 \mathrm{~cm}$ de ancho y la cúpula $3 \mathrm{~cm}$ de ancho. 
Muestra: CF 4600 (Fig. 7, a-d): La roca recolectada presenta una medida de $46 \mathrm{~cm}$ de largo por $12 \mathrm{~cm}$ de ancho. Se observan moldes de hojas con características de esta especie; son notorios 5 moldes de frutos de Quercus. Entre ellos resalta un molde de un fruto juvenil, donde se observa la nuez abriéndose paso entre la cúpula (Fig. 7b y Fig. 8b), presenta escamas muy conspicuas y su diámetro es de $2 \mathrm{~cm}$. Además, se observan cuatro cúpulas con notorias escamas y anchos variables entre 3,3 y $3,7 \mathrm{~cm}$. (Fig. 7, y Fig. 8a y 8c).

Muestra: CF 4595 (Fig. 9a): Molde de una cúpula, son notorias las escamas. Mide 3,4 $\mathrm{cm}$ de diámetro y un grosor aproximado de $2 \mathrm{~cm}$.

Muestra: CF 4596 (Fig. 9b): Molde de una cúpula, tiene claramente definido el pedúnculo y las escamas. Mide 3,3 cm de diámetro.

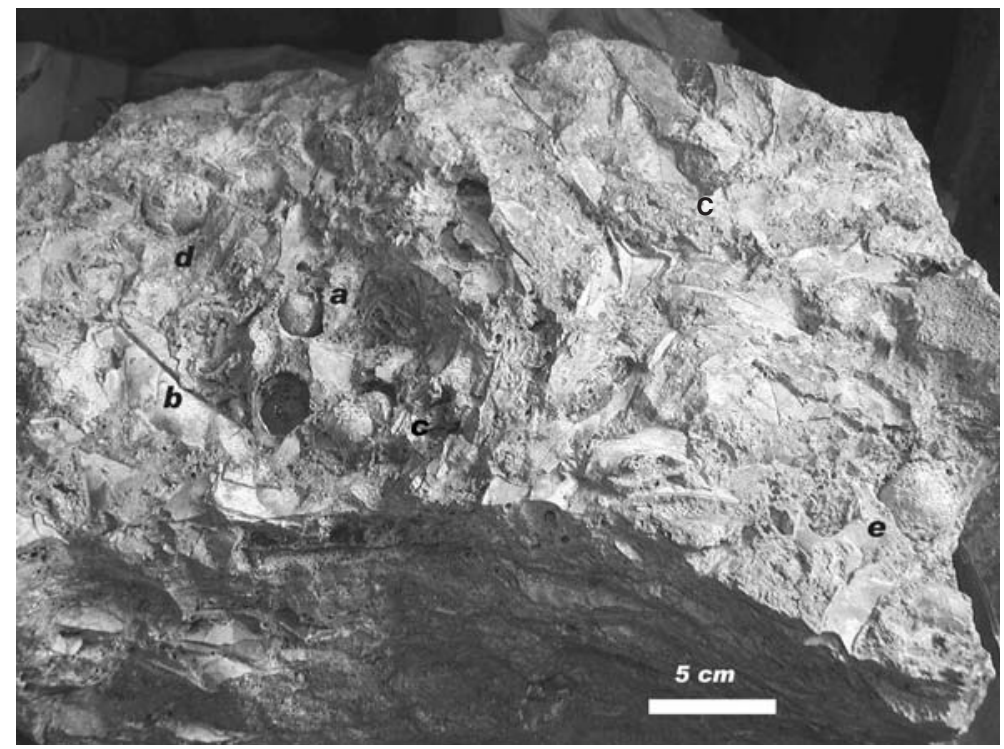

Fig. 3: CF 4598 - Roca con moldes de frutos y fragmentos de limbos fósiles; en la figura solo se aprecian $41 \mathrm{~cm}$ de largo de la roca, resaltándose 6 moldes de frutos (a,b,c (2 moldes), d y e).

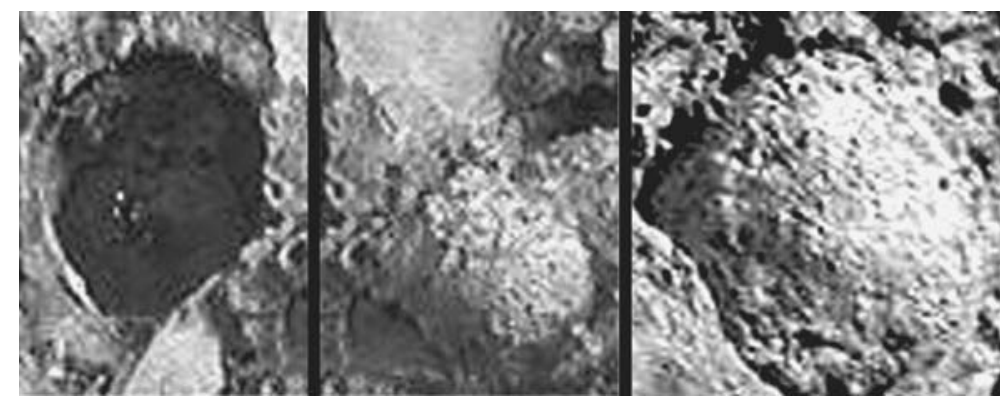

Fig. 4: Ampliaciones de los moldes b, c y e de la roca CF 4598.

Molde b: Concavidad de nuez, se observa en la parte superior impresiones de la cúpula. Mide 3,5 cm de alto x 2,5 cm de ancho. Molde c: Molde de dos frutos, la nuez superior muestra también parte de la cúpula. Mide aproximadamente de 2,5 cm de ancho. Molde e: Molde de nuez, mide aproximadamente 3,7 cm de diámetro. 


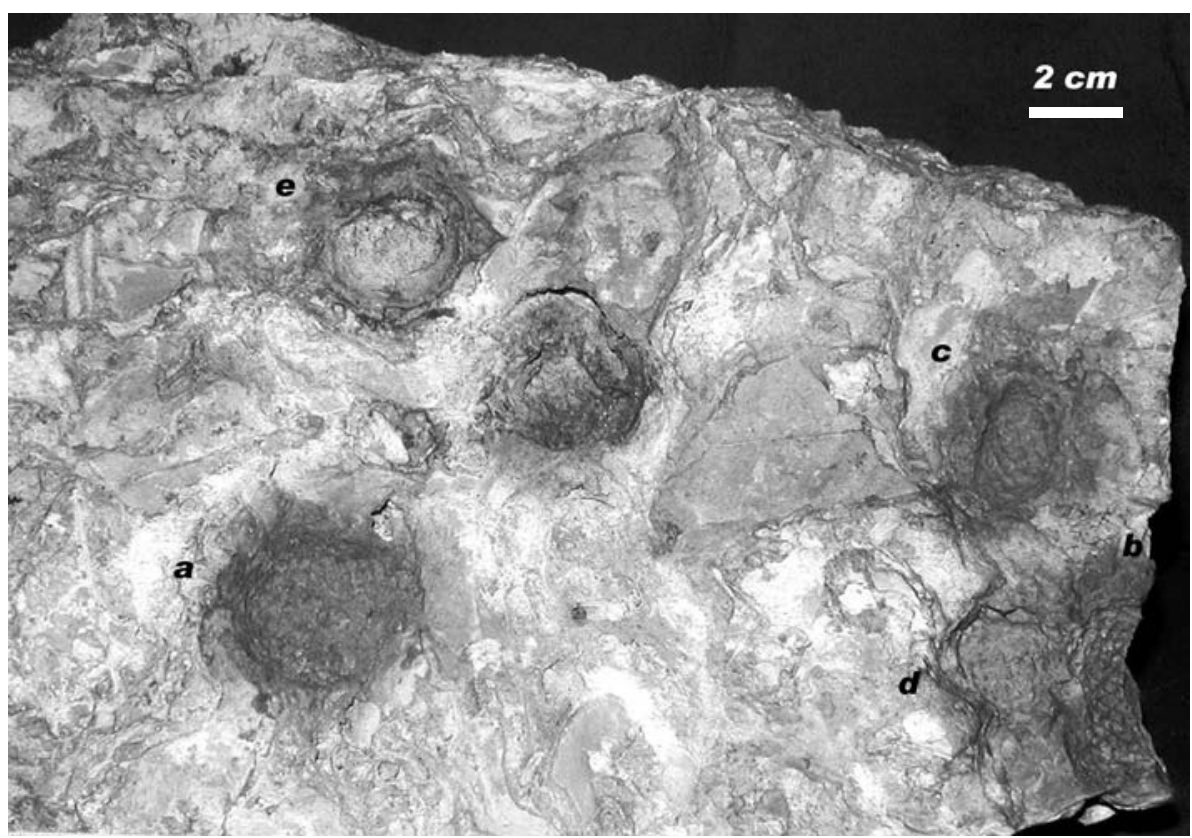

Fig. 5: CF 4599. En esta figura solo se muestran $25 \mathrm{~cm}$ de la roca, con los 5 moldes más visibles de frutos (a,b,c,d y e), también se observan bastantes moldes de limbos incompletos con las características de esta especie.

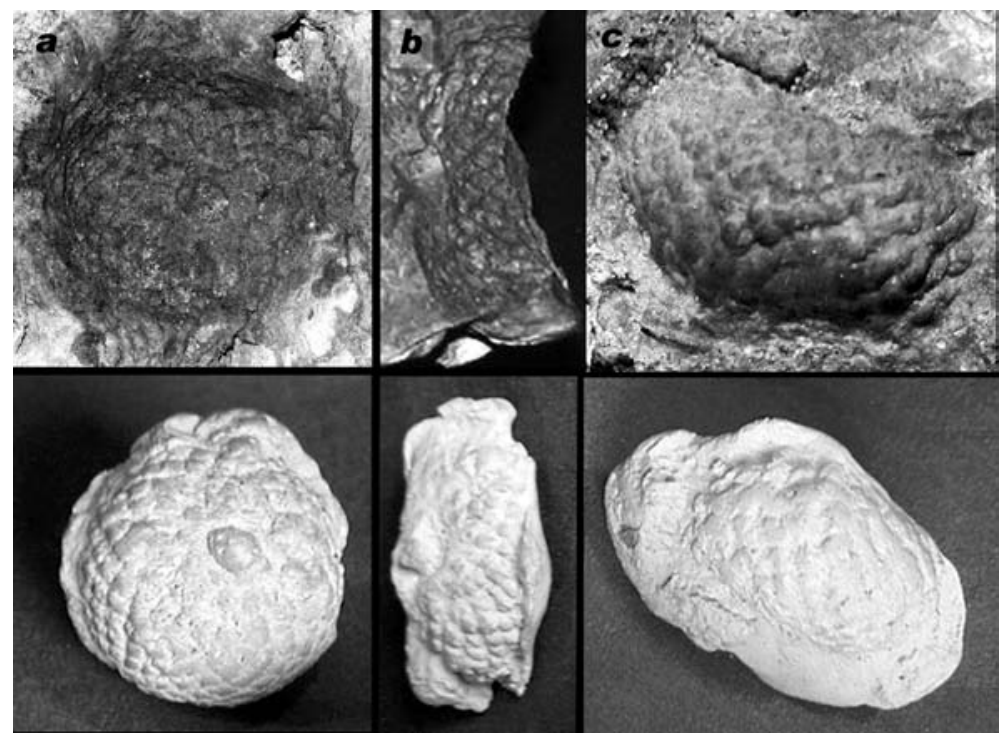

Fig. 6: Ampliaciones de los moldes y contramoldes de la roca CF 4599. Molde y contramolde a: En la parte superior un molde cóncavo de la cúpula de un fruto grande, se observa claramente en el centro la inserción del pedúnculo, así como las escamas que lo componen. En el contra molde realizado (inferior) también se ven claramente estas características. La cúpula tiene $4,5 \mathrm{~cm}$ de diámetro, con una altura de $2 \mathrm{~cm}$. Molde y contramolde b: En la parte superior un molde lateral de la cúpula de un fruto, tanto en éste como en el contra molde (inferior) se ven claramente las escamas y el pedúnculo. La medida lateral de la cúpula es de $4 \mathrm{~cm}$ de ancho. Molde y contramolde c: En la parte superior un molde lateral de la cúpula de un fruto pequeño, y en el contra molde (realizado) se ven claramente las escamas. El ancho de la cúpula es de 1,8 cm. 


\section{DISCUSIÓN}

Los registros de fósiles de este género, presentes en el país, corresponden a polen y fragmentos de troncos carbonizados.

Estudios palinológicos de la localidad de La Chonta (El Empalme) realizados por Hooghienstra et al. (1992), ubican Quercus dentro de los géneros ahí encontrados. Alvarado \& Leandro (1997) describen muestras de troncos fósiles como Quercus costaricensis? y Quercus copeyensis? Estos fragmentos de troncos carbonizados fueron recolectados en los sedimentos en el sitio del proyecto hidroeléctrico de Angostura. Fueron analizados por la bióloga Isabel Ma. Carpio M., en el Laboratorio de Productos Forestales del Instituto de Investigaciones en Ingeniería de la UCR, manifestando lo siguiente "...dicho material está muy deteriorado debido a que presenta pudrición, lo que hace sumamente difícil apreciar las características estructurales que son utilizadas para identificación.
Sin embargo, fue posible observar en algunas muestras, características que permiten compararlas con maderas que se encuentran en nuestras colecciones, permitiendo deducir que las siguientes especies podrían estar incluidas dentro de estos fósiles". Luego de un cuadro que resume las muestras encontradas en 5 familias, continúa la especialista mencionando además, que "Es importante tener presente que también podría tratarse de otras especies con características estructurales muy semejantes a las presentadas en el cuadro No. 1, por lo tanto debe presentarse el nombre científico con un signo de interrogación." (Alvarado \& Leandro, 1997).

Los moldes de frutos de Quercus corrugata procedentes de la Palmera de San Carlos corresponderían con el tercer registro de especímenes fósiles de este género, y el primero de esta especie.

Distribución biogeográfica actual: Quercus corrugata Hooker, se localiza desde Chiapas

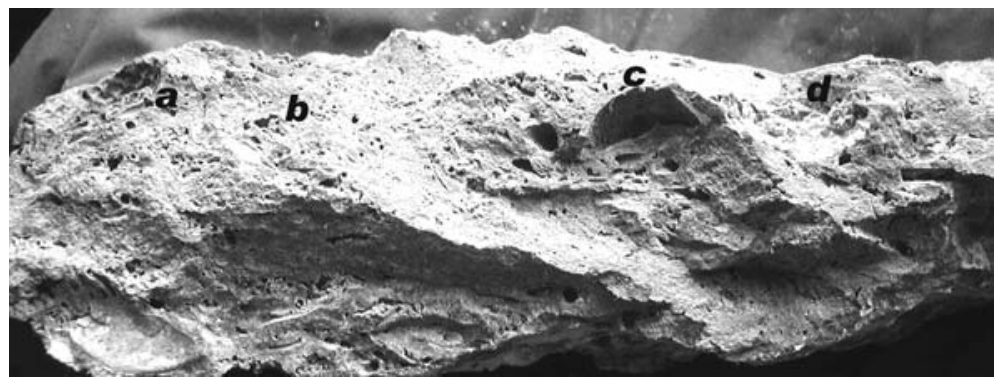

Fig. 7: CF 4600. La figura muestra solo $23 \mathrm{~cm}$ de la roca, en ella se observan moldes de hojas y de frutos. Solo se marcaron los 4 moldes más visibles.

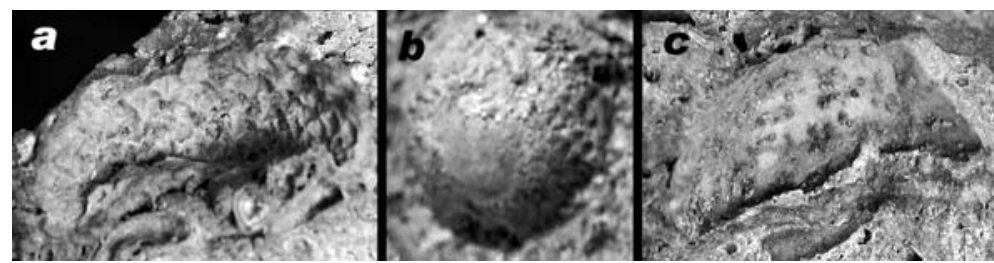

Fig. 8: Ampliaciones de los moldes a, b y c de la muestra CF 4600. Molde a: lateral de una cúpula, son notorias las escamas. Mide $3,7 \mathrm{~cm}$ de ancho. Molde b: un fruto juvenil, se observa la nuez abriéndose paso entre la cúpula que presenta claramente sus escamas. Mide $2 \mathrm{~cm}$ de diámetro. Molde c: una cúpula de fruto, mide $3 \mathrm{~cm}$ de ancho. 
en México, hasta la parte occidental de Panamá, desde los 1200 a los 2000 metros de altitud (Burger, 1977).

En Costa Rica la mayoría de los especímenes citados, se localizan en la vertiente pacífica, pero hay registro de especímenes en las montañas de Monteverde del lado de la vertiente atlántica (Haber, 1990).

Paleoclimatología: $Q$. corrugata, en la actualidad habita en bosques montanos. Su registro en el afloramiento de La Palmera solo puede ser explicada por una migración vertical de la vegetación asociada a un cambio climático en el pasado. Es muy probable que esta migración ocurriese durante el Pleniglacial del Pleistoceno (50 000 a 13000 años AP).

Esta hipótesis parece corroborarse con la información palinológica estudiada por Hooghienstra et al. (1992), obtenida en la localidad de La Chonta en la carretera interamericana sur. Hooghienstra et al. (1992) concluyen que el límite arbóreo superior (que corresponde con el bosque montano bajo: el robledal de Quercus con el bambú Chusquea, bajó por enfriamiento de la región casi $800 \mathrm{~m}$. No se tienen datos de cuanto cayó el límite inferior del bosque montano. De acuerdo con esta información, cabe esperar que la base del ámbito de $Q$. corrugata que actualmente es de 1200 msnm cayera al menos $800 \mathrm{~m}$ (en relación con el nivel actual), ubicándose a los $400 \mathrm{msnm}$. Esto explicaría la presencia de frutos fósiles en la localidad de la Palmera, pues este ámbito de altura concuerda con los puntos más altos de los afloramientos de travertino. Por ello, este registro debe ser considerado un indicador paleoclimático de temperatura para la localidad de La Palmera.

Edad: Es posible extrapolar la edad del registro de $Q$. corrugata de La Palmera, a partir de los datos palinológicos de la turbera de La Chonta, obtenidos por Hooghiemstra et al. (1992), Islebe (1996) e Islebe et al. (1995 y 1996), resumidos en Kappelle (1996). Dichos autores registraron que "durante el Pleniglacial (ca. 50000 a 13000 años AP), el límite superior del bosque bajó hasta casi $2000 \mathrm{msnm}$ (desde los $2800 \mathrm{~m}$ ) por enfriamiento". El máximo enfriamiento y la máxima caída del límite arbóreo superior, equivalente a $800 \mathrm{~m}$, ocurrió de acuerdo al gráfico de Islebe (en Kappelle, 1996) alrededor de los 18000 años antes del presente, por lo que la edad más probable del yacimiento de La Palmera puede asumirse como Pleistoceno Superior.

\section{CONCLUSIONES}

Se recuperaron 21 moldes de nueces, que se identificaron como pertenecientes a la especie Quercus corrugata Hook, de la familia Fagacea, conocidos como robles de montaña. Es la primera

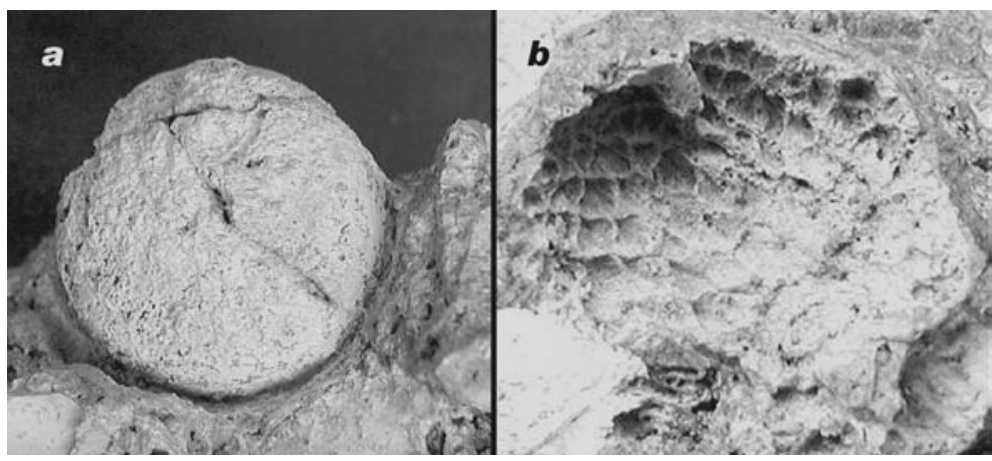

Fig. 9: a: CF 4595. Molde de una cúpula, son notorias las escamas mide 3,4 cm de diámetro y un grosor aproximado de $2 \mathrm{~cm}$. b: CF 4596. Molde de una cúpula, tiene claramente definido el pedúnculo y las escamas. Mide 3,3 cm de diámetro. 
vez que se registran frutos de esta especie como fósil en Costa Rica y América Central.

La caída regional de la base del bosque montano durante el Pleniglacial del Pleistoceno, permitió la colonización de bosques de Quercus corrugata en alturas de alrededor de $400 \mathrm{msnm}$. Es posible concluir que al finalizar el último período glacial, la distribución de Quercus corrugata, en nuestras latitudes, se restringió en áreas montanas sobre los $1200 \mathrm{~m}$ y $1900 \mathrm{~m}$.

Una edad probable de la localidad de La Palmera, fundamentada en este hallazgo, corresponde con el Pleistoceno Superior.

\section{REFERENCIAS}

ALVARADO, G. \& LEANDRO, C., 1997: Actualización geológica-geofísica de los sitios en donde se construirán las grandes obras del P.H. - Angostura: un enfoque hacia problemas ingenieriles conexos. -88 págs. ICE [Informe inédito].

BURGER, W., 1977: Fagacea. - En: BURGER, W. (ed.): Flora Costaricensis. - Ann. Fieldiana Bot. 40: 59-82.
HABER, W., 1990: Lista provisional de las plantas de Monteverde, Costa Rica. - Brenesia, 34: 63-120 [1991].

HOOGHIEMSTRA, H., CLEEF, A.M., NOLDUS, G.W. \& KAPPELLE, M., 1992: Upper Quaternary vegetation dynamics and palaeoclimatology of the La Chonta bog area (Cordillera de Talamanca, Costa Rica). - Quaternary Science, 7(3): 205-225.

KAPPELLE, M., 1996: Los bosques de robles (Quercus) de la Cordillera de Talamanca, Costa Rica. - 336 págs. Ed. Inbio-Universidad de Amsterdam.

MONTIEL, L. M., 1994: Introducción a la flora de Costa Rica. - 346 págs. Edit. UCR, San José.

PÉREZ, E. A., 2001: Comparación de la flora fósil del pleistoceno con la flora actual en la localidad de la Palmera de San Carlos provincia de Alajuela. - 126 págs. Escuela de Ciencias Exactas y Naturales, Programa de licenciatura en Manejo de Recursos Naturales. Univ. Est. Distancia [Tesis Lic.]. 